\title{
Estado de la salud bucal en el Ecuador
}

\section{Oral health status in Ecuador}

\section{Resumen}

En el Ecuador se presenta una alta prevalencia e incidencia de diferentes patologías bucales, este escenario es similar en muchos países de la región. Las enfermedades orales constituyen un problema de salud pública, debido a que requiere un alto costo en su manejo y control; es por ello que se ha generado énfasis en realizar actividades preventivas que permitan mejorar el panorama. Han sido varias las medidas adoptadas por entes gubernamentales en el Ecuador para reducir la incidencia principalmente de caries dental, desde la prevención masiva a través de la fluoración de diferentes elementos de consumo humano, la promoción y prevención de salud oral a través de la enseñanza de técnicas de cepillado dental, difusión del correcto uso de seda dental, detección temprana de placa bacteriana, charlas educativas motivadoras y colocación de sellantes en superficies oclusales de molares definitivos en escolares de poblaciones urbano-marginales y rurales. Sin embargo, dichas estrategias no han conseguido ser monitoreadas, evaluadas ni reportadas para conocer su efectividad en la mejora de la salud oral en nuestro país.

Palabras clave: Ecuador, salud bucal, Odontología en salud pública, epidemiología (fuente: DeCS BIREME).

\begin{abstract}
In Ecuador, there is a high prevalence and incidence of different oral pathologies, this scenario is similar in several countries in the region. Oral diseases constitute a Public Health issue, since they involve a high investment in their management and control. Thus, an emphasis in preventive activities has been placed to improve the impact of oral diseases. Several measures have been taken by the Ecuadorian government to reduce the incidence of dental caries, including massive prevention programmes. For instance, fluoridation of food and water, school teaching of right tooth brushing techniques and dental floss use, early detection of bacterial plaque, delivery of educational talks, and sealant placement on occlusal surfaces of permanent teeth in schoolchildren from deprived urban and rural populations. Nevertheless, these strategies have not been monitored,
\end{abstract}

\section{Nota Científica}

\author{
Juan Marcos Parise-Vasco 1,2,a, Paula Zambrano-Achig 1,2,a, \\ Andrés Viteri-García ${ }^{1,2, b}$, Ana Armas-Vega ${ }^{1, c}$ \\ 1 Universidad UTE, Facultad de Ciencias de la Salud \\ "Eugenio Espejo", Quito, Ecuador. \\ ${ }^{2}$ Centro de Investigación en Salud Pública y Epidemiología \\ Clínica (CISPEC), Quito, Ecuador. \\ a Odontólogo.

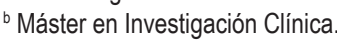 \\ ${ }^{c} \mathrm{PhD}$ en Operatoria Dental.
}

\section{Correspondencia:}

Ana Armas-Vega: ana.armas@ute.edu.ec Universidad UTE, Facultad de Ciencias de la Salud

"Eugenio Espejo". Av. Mariana de Jesús S/N y Av. Mariscal Sucre, Quito, Ecuador. Código postal: 170147

ORCID: 0000-0003-3800-8166

\section{Coautores:}

Juan Marcos Parise-Vasco: juan.parise@ute.edu.ec ORCID: 0000-0002-5223-3370

Paula Zambrano-Achig: paula.zambrano@ute.edu.ec ORCID: 0000-0003-3632-4296

Andrés Viteri-García: andres.viteri@ute.edu.ec ORCID: 0000-0003-0393-2404

\section{Editora invitada:}

Rosa Ana Melgar Hermoza

Universidad Peruana Cayetano Heredia, Perú.

Conflicto de intereses: los autores declaramos no tener conflictos de interés.

Fuente de financiamiento: autofinanciado

Recibido: 08/04/20

Aceptado: $14 / 04 / 20$

Publicado: 04/08/20 
evaluated or reported in order to understand their success in improving the Oral Health status of our country.

Keywords: Ecuador, oral health, public health dentistry, epidemiology (source: MeSH NLM).

\section{Introducción}

Las enfermedades orales son consideradas un problema de salud pública a nivel mundial, con una alta prevalencia en países de bajos y medianos recursos, pero sobre todo de un gran impacto económico asociado a su manejo ${ }^{1,2}$. Si bien de forma general las patologías bucales son de naturaleza crónica y progresiva, afectan a toda la población a lo largo de su vida, existen grupos que por sus condiciones económicas y sociales pueden ser considerados de alta susceptibilidad ${ }^{3}$.

La caries dental, enfermedad periodontal y maloclusión son patologías consideradas prevalentes en países de primer mundo así como en países de bajos recursos ${ }^{4}$. La caries dental está presente en alrededor del 60\% de la población, lo que demuestra que los esfuerzos ejecutados hasta el momento, aún no consiguen intervenir en los factores desencadenantes de la enfermedad, como el elevado consumo de azúcares y la exposición inadecuada de fluoruros ${ }^{2,5}$, situación que se muestra más compleja a nivel de países de nuestra región, donde la prevalencia es mayor al $80 \%{ }^{6}$.

\section{Estado de la salud bucal en Ecuador}

La situación de la salud bucal del Ecuador se ha descrito en un estudio epidemiológico realizado en 1996 y un reporte realizado por Raza el 2009, ambos estudios demuestran la elevada prevalencia de los problemas bucales ${ }^{7,8}$. El reporte de 1996 demuestra la prevalencia de caries dental en escolares de 6 a 15 años en $88,2 \%$, para 2009 la prevalencia de caries dental en los escolares examinados en ese mismo rango etario disminuyó a 75,6\% ${ }^{7,8}$. Estudios más recientes en muestras poco representativas han reportado una prevalencia del $70 \%$ de caries en niños de 8 a 10 años 9,10 .

Las enfermedades periodontales constituyen un grupo de patologías prevalentes, con una frecuencia del $20 \%$ al $50 \%$ de la población, que aumenta con la edad, observando una frecuencia del $70 \%$ de los adultos mayores de 65 años, con una frecuencia de $56,4 \%$ en el grupo masculino ${ }^{11,12}$. Esta patología se ha asociado a factores relacionados a higiene, consumo de cigarrillo, enfermedades sistémicas como la diabetes, consumo de varios medicamentos y la edad considerados como factores de incremento de la enfermedad periodontal ${ }^{13}$; en el reporte del estudio epidemiológico de 1996, donde se valoró a escolares de 12 a 15 años, $65,2 \%$ presentaron la enfermedad, se mostró una reducción al obtener el $10,4 \%$ en sujetos del mismo grupo etario en el estudio del $2009^{7,8}$.

La maloclusión dental es considerada también una de las patologías con mayor prevalencia, esta afecta a los tejidos duros y bandos, alrededor de la cavidad oral y la articulación temporomandibular, con una prevalencia de un $20 \%{ }^{14}$. En el Ecuador el estudio de Ruiz (1996) reportó en escolares de 12 a 15 ańos un 35\% de prevalencia de maloclusión considerada como leve y un 13,5\% de maloclusiones moderadas y severas ${ }^{7}$. Varios estudios realizados con poblaciones pequeñas en diferentes ciudades del Ecuador reportaron porcentajes de maloclusiones del $29 \%$ a $70 \%$ en Clase I, valores de $10 \%$ a $34 \%$ Clase II y de entre $10 \%$ a $19 \%$ Clase III, considerando individuos en edades entre 20 a 27 años 15,16,17,18.

La fluorosis también fue valorada en los dos estudios ejecutados en el país, en escolares de 12 a 15 años, reportando un $5 \%$ de prevalencia ${ }^{7}$. Además, se reportó la necesidad de tratamiento odontológico en un $14,8 \%$ en escolares de entre 6 a 15 años ${ }^{8}$, donde la frecuencia de procedimientos de operatoria alcanzo un $85,2 \%$, exodoncias un $16,9 \%$ y endodoncia un $10,5 \%{ }^{19}$.

\section{Políticas y estrategias para mejorar la salud bucal aplicadas en el Ecuador}

El derecho a la salud en el Ecuador fue reconocido por la Constitución Política de 1979, sin embargo, gobiernos anteriores realizaron esfuerzos por implementar leyes para garantizar el acceso a los servicios de salud ${ }^{20}$, motivando el 16 de junio de 1967 la creación del Ministerio de Salud Pública del Ecuador ${ }^{21}$; institución que, en el año de 1970, estableció la obligatoriedad para que los egresados de odontología presten sus servicios profesionales como requisito previo a la inscripción del título profesional, a través del "Plan Nacional de Salud Rural". A partir del año 1972, fue creado el Departamento de Odontología Rural y la Comisión Nacional de Odontología, entidad encargada de elaborar el Plan de Desarrollo Integral de la Odontología, enfatizando la promoción de servicios y producción de conocimientos mediante actividades de investigación, planificación, capacitación, monitoreo y evaluación en esta área ${ }^{22}$.

Para 1973, fue creada la División Nacional de Odontología, entidad que consiguió la asignación presupuestaria para el desarrollo de los programas orientados a proporcionar agua potable fluorada, como refuerzo de programas de educación y prevención en comunidades marginadas ${ }^{23}$. En 1974, se establece en Ecuador mediante Decreto Supremo, el "Programa Nacional de Fluoración" del agua, mismo que fue ejecutado en varias ciudades del país hasta el año 1986, su implementación se dificultó por la baja cobertura de los sistemas de agua potable, especialmente en zonas rurales ${ }^{24}$.

En 1986, se crea la Dirección Nacional de Estomatología, ente rector de la odontología pública en Ecuador hasta el año $2003^{23}$, quien para 1988, realiza un estudio epidemiológico de salud bucal en niños de escuelas fiscales del país, donde se refleja una alta prevalencia e incidencia de caries, periodontopatías y maloclusiones 
${ }^{19}$; indicadores que redefinieron la problemática odontológica, obligando a la Dirección Nacional de Estomatología a planificar de acuerdo a una realidad objetiva ${ }^{25}$. En 1992, fue publicado el "Manual de Normas Estomatológicas para el Primer Nivel de Atención", con la asesoría de la OPS/OMS y para 1995 se crea el "Manual de Normas y Procedimientos Estomatológicos", con el objetivo de establecer lineamientos y unificar criterios en la atención y prevención de las diferentes patologías bucales ${ }^{23,25}$.

Como alternativa para la prevención masiva de caries dental, en 1996, se implementó mediante Decreto Ejecutivo el "Programa Nacional de Fluoración de la Sal de Consumo Humano", instaurando de forma obligatoria la incorporación de yodo y flúor en la sal producida en el país, estableciéndose como norma técnica la adición de flúor en forma de fluoruro de sodio o de potasio, en cantidades de 200 a 250 ppm; excluyendo su distribución a localidades donde los niveles de flúor en agua de consumo humano se encuentren mayores a $0,7 \mathrm{ppm}$ ${ }^{26}$. Se tomó como criterio el estudio de línea basal, ejecutado en el mismo año, sobre el "Contenido Natural de Flúor en los Abastecimientos de Agua de Consumo Humano del Ecuador", se realizó posteriormente un "Estudio Epidemiológico de Salud Bucal en Escolares Fiscales menores de 15 años del Ecuador", con el fin de tener datos nacionales para medir el impacto del programa a mediano y largo plazo ${ }^{24,7}$, dichos estudios no se han realizado.

En el año 2008, se aprobó la actual Constitución Política del Ecuador, que expresa a través de los artículos $32,358,359,360,363$, que la salud es un derecho fundamental y el Estado está encargado de garantizarla a través del sistema nacional de salud ${ }^{27}$. Además, la Ley Orgánica de Salud establece en su artículo 4 que "La autoridad sanitaria nacional es el Ministerio de Salud Pública...", y en su artículo 6, refiere ser su competencia "...diseñar e implementar programas de atención integral y de calidad a las personas durante todas las etapas de la vida y de acuerdo con sus condiciones particulares..." 28,29 .

Para cumplir estas legislaciones, en el año 2009, la Secretaría Nacional de Planificación y Desarrollo (SENPLADES) elaboró el Plan Nacional de Desarrollo para El Buen Vivir, documento que sirvió de base para la creación del Modelo de Atención Integral Familiar, Comunitario e Intercultural de Salud (MAIS-FCI), publicado en 2011, con carácter de implementación obligatoria en los establecimientos de todos los niveles de atención del Sistema Nacional de Salud, con el propósito de reorientar el enfoque curativo, centrado en la enfermedad y el individuo, hacia un enfoque centrado en la promoción, prevención y cuidado integral de la salud ${ }^{20,30}$.

Si bien el MAIS-FCI no nombra ni establece funciones al odontólogo en el equipo de atención integral de salud, la estomatología es mencionada únicamente en su anexo $2{ }^{29}$; el "Plan Nacional de Salud Oral" desarrollado en 2009, sirve como complemento a este documento y atribuye al odontólogo realizar prestaciones de promoción y prevención en salud bucal ${ }^{23,30}$, que complementaron las acciones ya establecidas años atrás, basadas en acciones de promoción, enseñanza de las técnicas de cepillado dental, uso del hilo dental, detección de placa bacteriana a través charlas educativas dirigidas tanto a padres de familia, maestros y escolares ${ }^{31}$.

En el 2018, se redactó el "Manual de Atención Integral de Salud en Contextos Educativos (MAIS-CE), desarrollado como complemento al MAIS-FCI, el cual prioriza proteger la salud de la población estudiantil, y considera a la atención odontológica como eje fundamental en el cuidado integral de la salud, estableciendo que el personal odontológico debe realizar actividades como: levantamiento del odontograma, detección temprana de malos hábitos bucales y registro del índice de dientes cariados, perdidos y obturados (CPO y ceo) por niño, así como el seguimiento a los casos de niños con índices más alto de prevalencia de caries, detección de placa bacteriana, profilaxis, aplicación de flúor y aplicación de sellantes; se enfatiza la prevención de las enfermedades bucodentales a nivel masivo, grupal e individual a través del mejoramiento de hábitos higiénico-alimentarios, eliminación de hábitos nocivos y a la conservación de piezas dentales sanas ${ }^{23,32}$. En este modelo, el Ministerio de Salud Pública es el encargado de proveer todos los medicamentos, insumos y equipos odontológicos a través del establecimiento de salud del primer nivel de atención al que está adscrito la institución educativa ${ }^{32}$.

Resulta evidente el énfasis en acciones preventivas en las políticas de salud pública sobre todo en beneficio de grupos prioritarios en poblaciones urbano-marginales y rurales a través de programas y servicios en forma gratuita; sin embargo, resulta imperativo conocer si estas medidas y esfuerzos implementados por el Ministerio de Salud Pública han sido efectivas, por lo cual se sugiere realizar un nuevo estudio epidemiológico nacional con el fin de medir la incidencia y prevalencia de las diferentes enfermedades bucales, y conocer si estas han disminuido en comparación con el último registro ${ }^{29}$.

\section{Lecciones aprendidas y retos futuros}

Los estudios epidemiológicos realizados en el Ecuador 7,8 evidencian el incremento del número de lesiones cariosas en relación directa con el aumento de la edad del individuo. Indicador que revela fallas en el enfoque y direccionamiento de las acciones aplicadas por parte de los organismos encargados de la salud. A pesar de que los reglamentos ecuatorianos garantizan el acceso a los servicios de salud ${ }^{20}$, sobre todo a las poblaciones más vulnerables y se han realizado varios esfuerzo por ampliar el número de actividades de prevención, en su gran mayoría el porcentaje de actividades en salud pública se limitan a acciones curativas.

En el área estomatológica, el Ministerio de Salud Pública del Ecuador a través del "Plan Nacional de Salud" fomentó la promoción de servicios y producción de conocimientos mediante actividades de investigación, planificación, capacitación, monitoreo y evaluación en esta área ${ }^{22}$. Sin embargo, la promoción de servicios se 
ha limitado a la prestación de servicios a través de programas que incluyen acciones curativas dirigidas principalmente a la población escolar. Actividades que se encuentran fuera del enfoque odontológico actual de prevención y que exigen un alto presupuesto, debido al empleo de materiales odontológicos adhesivos costosos e importados. Esta problemática se agudiza por el empleo de personal de salud que muchas veces se encuentra desactualizado en conocimiento o sobrecargado de responsabilidades.

En esta misma línea de pensamiento, la producción de conocimientos con actividades vinculadas a investigación, es una de las metas de las entidades de gobierno en los distintos programas desarrollados, que no se han logrado alcanzar. Se atribuye esta problemática a la falta de presupuesto, ausencia de personal calificado y debidamente capacitado, pero, sobre todo, al poco interés para su ejecución por parte de las autoridades responsables. Frente a esta realidad, los gremios de profesionales y la academia han empezado a realizar estudios para comprobar la efectividad de lo ejecutado por las entidades de gobierno en comparación con los resultados del último estudio epidemiológico realizado en el país, lamentablemente sin contar con un resultado conclusivo. Factores que nos hacen percibir que el cumplimiento de las metas propuestas por la OPS/OMS ${ }^{34}$ son una utopía.

De igual forma, la incorporación de flúor promovido como estrategia de salud a nivel mundial ${ }^{25}$, implementada en su momento en el Ecuador, no se ha monitoreado en cuanto a su principal función, por lo que se carece de datos que evidencien su eficacia en la prevención de caries dental en esta población, sin embargo, sirvieron para que los gobiernos seccionales realicen monitoreo de sus aguas de consumo, lo que realmente ha representado una ventaja en cuanto al establecimiento de medidas de salud pública.

En los últimos años, múltiples campañas encabezadas por empresas privadas responsables de la comercialización de productos de higiene oral, han volcado sus esfuerzos a promover la prevención a través del cepillado dental y el uso controlado de pasta dental. Sin existir aún resultados que estimulen su ejecución. La historia revela pobres resultados cuando las acciones son ejecutadas desde un solo frente. La evidencia científica demuestra la necesidad de establecer acciones preventivas integrales enfocadas y específicamente dirigidas a un grupo etario determinado ${ }^{35}$; acciones que permitan alcanzar el equilibrio de bienestar a la población, acompañadas del progreso científico y tecnológico que la odontología sufre constantemente; ancladas a profesionales odontólogos preparados, motivados y entrenados de manera integral en la ejecución de técnicas y procedimientos pero sobre todo conscientes de que el estado bucal constituye un reflejo de la salud del individuo y de que, la caries dental es aún la lesión más frecuente que afecta sin distinción a los diferentes grupos etarios y económicos.

El diagnóstico oportuno, será siempre la estrategia más adecuada y la principal acción a realizarse, la delegación de responsabilidades a los gobiernos nacionales o seccionales, o entes de control sanitario, no ha producido beneficio alguno hasta el momento. Lastimosamente tendremos que dejar como herencia a las nuevas generaciones de odontólogos, los sueńos de cumplimiento de las metas establecidas en la búsqueda de mejoras en la salud de nuestros pacientes. Nos queda a esta generación la satisfacción de haber conseguido concientizar al paciente sobre la importancia de un cambio de hábitos. Los errores cometidos, tendrán que convertirse en el abono que permita a la semilla dejada entre los profesionales, que constituyen el relevo generacional, germinar, vertiendo sobre éstos, la conciencia de la constante necesidad de un enriquecimiento científico basado en evidencias probadas.

\section{Conclusiones}

El Ecuador se ha esforzado en aplicar diversas estrategias en la búsqueda de la reducción en la incidencia de la caries dental, no obstante, las acciones ejecutadas hasta el momento no han podido recibir seguimiento. Una fortaleza de las políticas actuales es la promoción y prevención a través de la enseñanza de técnicas de cepillado dental, detección temprana de placa bacteriana y charlas educativas motivadoras; actividades que se muestran como base fundamental para alcanzar las metas planteadas por los organismos de salud internacionales. Sin embargo, aún existe mucho camino por recorrer, y será el cambio de pensamiento de los futuros profesionales de la salud lo que permita alcanzar lo propuesto años atrás.

\section{Agradecimientos}

Agradecemos a la MSc. Camila Montesinos, docente de la Universidad UTE, por la traducción del resumen de este artículo al idioma inglés.

\section{Referencias bibliográficas}

1. Kassebaum N, Smith A, Bernabé E, Fleming T, Reynolds A, Vos T, et al. Global, regional, and national prevalence, incidence, and disability-adjusted life years for oral conditions for 195 countries, 1990-2015: A systematic analysis for the global burden of diseases, injuries, and risk factors. J Dent Res. 2017;96(4):380-7.

2. Petersen PE, Bourgeois D, Ogawa H, Estupinan-Day S, Ndiaye C. The global burden of oral diseases and risks to oral health. Bull World Heal Organ. 2005;83(9):661-9.

3. Petersen PE. The World Oral Health Report 2003: continuous improvement of oral health in the 21 st century - the approach of the WHO Global Oral Health Programme. Community Dent Oral Epidemiol. diciembre de 2003;31(s1):3-24.

4. World Health Organization. Oral health [Internet]. [citado 25 de marzo de 2020]. Disponible en: https://www. who.int/news-room/fact-sheets/detail/oral-health

5. Masson M, Simancas-Racines D, Viteri-García A. La industria de alimentos y bebidas azucaradas y las instituciones de Investigación en Odontología. ¿Conflictos de interés? Rev Ecu Med Eugenio Espejo. 2019 (7)12 
6. Aquino Canchari CR. Prevalencia, experiencia y significancia de caries dental en escolares de Cutervo, Perú, 2017. Rev Odontol Act. 2018;3(2):21-4.

7. Ruiz O, Narváez A, Narváez E, Herdoíza M, Torres I, Pinto G, et al. Estudio Epidemiológico de Salud Bucal en Escolares Fiscales menores de 15 años. Ministerio de Salud Pública del Ecuador. Quito; 1996.

8. Raza X, Alvear A, Andrade R, Ayala E, Chiliquinga M. Estudio Epidemiológico Nacional de Salud Bucal en Escolares Menores de 15 ańos de Ecuador 2009-2010. Boletín Informativo OPS. 2011.

9. Jarrín-Peñafiel MJ, Toalombo-Puma OV, Carrera-Robalino AE, Quintana-Carrillo EA, Ribadeneira-Morales LA, Armas-Vega A. Riesgo cariogénico y su relación con el factor socio económico, en niños de 8-10 años de una zona agrícola del Ecuador. Rev Cient Odontol. $2018 ; 14(1)$.

10. Cabeza-Bernhardt G, González-Andrade F, Paredes-Andrade C. Estado de salud oral en el Ecuador. Rev Odontol Act. 2016;1(3):65-70.

11. Centers dor Disease Control and Prevention CDC. Periodontal Disease [Internet]. [citado 25 de marzo de 2020]. Disponible en: https://www.cdc.gov/oralhealth/ conditions/periodontal-disease.html

12. Tonetti MS, Jepsen S, Jin L, Otomo-Corgel J. Impact of the global burden of periodontal diseases on health, nutrition and wellbeing of mankind: A call for global action. J Clin Periodontol. 2017;44(5):456-62.

13. Nazir MA. Prevalence of periodontal disease, its association with systemic diseases and prevention. Int J Health Sci (Qassim). 2017;11(2):72-80.

14. World Oral Health. Salud bucodental [Internet]. [citado 25 de marzo de 2020]. Disponible en: https://www.who. int/es/news-room/fact-sheets/detail/oral-health

15. López-Gallegos E, Armas A, López-Ríos E, Nuñez A, Tatés-Almeida K. Prevalencia de hábitos deletéreos y maloclusiones en dentición mixta en niños de la ciudad de Quito, Ecuador. Kiru. 2018;15(3):121-6.

16. Avellán-Arguello K. Maloclusiones dentales como factor de riesgo de trastornos temporomandibulares en pacientes de UCSG 2017. Rev Científica «Especialidades Odontológicas UG». 2017;117(1).

17. Salinas-Abarca L, Urgiles-Urgiles C, Jiménez-Romero M. Malocolusiones dentales en escolares de 12 ańos en la parroquia el Sagrario-Cuenca 2016. Rev Salud Vida Sipanense. 2017;4(2):58-66.

18. Parise-Vasco J, Villarreal-Ortega B, Zambrano-Achig P, Armas-Vega A, Viteri-García A. Prevalencia de maloclusiones en estudiantes de la carrera de Odontología de la Universidad UTE. De próxima aparición 2020.

19. Ruiz O, Solís H. Estudio epidemiológico de salud bucal en escolares del Ecuador. Ministerio de Salud Pública del Ecuador. Quito; 1988.
20. Jiménez-Barbosa W, Granda-Kuffo M, Ávila-Guzmán D, Cruz-Díaz L, Flórez-Parra J, Mejía L, et al. Transformaciones del Sistema de Salud Ecuatoriano. Rev Univ Salud. 2017;19(1):126-39.

21. Ministerio de Salud Pública del Ecuador. Protocolos odontológicos. 1. ${ }^{a}$ ed. Quito: Programa Nacional de Genética y Dirección Nacional de Normatización; 2013.

22. Palomeque P. Desarrollo de la odontología en el Ecuador, periodo 2008 - 2016. Espirales Rev Multidiscip Investig. 2018;2(13):35-48.

23. Ayala E, Pinto G, Raza X, Herdoíza M. Plan Nacional de Salud Bucal. Ministerio de Salud Pública del Ecuador. Quito; 2009.

24. Ruiz O, Narváez E, Pinto G, Raza X, Herdoiza M, Torres I, et al. Estudio del contenido natural de fluor en el agua de consumo humano de los abastecimientos del Ecuador. Ministerio de Salud Pública del Ecuador. Quito: Dirección Nacional de Estomatología; 1996.

25. Ayala E, Herdoíza M, Pinto G, Raza X. Normas y procedimientos de atención en salud bucal - Primer Nivel. Ministerio de Salud Pública del Ecuador. Quito; 2009.

26. Durán-Ballén S. Reglamento de la ley de yodación de la sal para consumo humano. Registro Oficial 998. Quito, Ecuador; 1996.

27. Constitución de la República del Ecuador. Registro Oficial 449. Qutio, Ecuador; 2008.

28. "Ley Orgánica de Salud". Registro Oficial 423. Quito, Ecuador; 2006.

29. Masson M, Simancas-Racines D, Viteri-García A. Salud oral en el Ecuador. Perspectiva desde la salud pública y la bioética. Práctica Fam Rural. 2019;4(3):24-7.

30. Ministerio de Salud Pública del Ecuador. Manual del Modelo de Atención Integral del Sistema Nacional de Salud Familiar Comunitario e Intercultural (MAISFCI). Quito; 2012.

31. Raza X, Pinto G, Ayala E. Manual de educación para la salud bucal para maestros y promotores. Ministerio de Salud Pública - Área de Salud Bucal. Quito; 2010.

32. Ministerio de Salud Pública del Ecuador. Manual de Atención Integral en Salud en Contextos Educativos (MAIS-CE). Dirección Nacional de Normatización. Quito; 2018.

33. World Health Organization. Oral health surveys : basic methods. 4. ${ }^{a}$ ed. Ginebra: World Health Organization; 1997.

34. Jordan AR, Becker N, Jöhren HP, Zimmer S. Early Childhood Caries and Caries Experience in Permanent Dentition: A 15-year Cohort Study. Swiss Dent J. 2016;126(2):114-9. 
